\title{
Splenic Infarction in a Postpartum Patient with COVID-19
}

\author{
Syed Nazeer Mahmood ${ }^{1}$ Yaser T. Dawod ${ }^{1} \quad$ Chee Man Chan $^{1}$
}

\author{
1 Section of Pulmonary/Critical Care, Department of Medicine, \\ MedStar Washington Hospital Center, Washington, District of \\ Columbia, United States
}

TH Open 2021;5:e81-e83.
Address for correspondence Syed Nazeer Mahmood, MD, Section of Pulmonary/Critical Care, Department of Medicine, MedStar Washington Hospital Center, 110 Irving St NW, Suite 2A50, Washington, DC 20010, United States (e-mail: snazeerm@gmail.com).
Thrombotic events have been reported in patients with coronavirus disease 2019 (COVID-19). Pregnant and postpartum patients are also known to be hypercoagulable and those with COVID-19 may therefore be at an even higher risk of thrombotic complications. Here, we discuss a postpartum patient with COVID-19 who developed an isolated splenic infarction. While describing a rare thrombotic event, this case serves to alert physicians to the higher risk of thrombosis in pregnant or postpartum patients with COVID-19.

A previously healthy 27-year-old female who was 37 weeks pregnant, with no past medical history presented to the hospital with complaints of fever, cough, and shortness of breath. She tested positive for COVID-19 and as she was improving, was discharged home. She presented to the hospital again 2 days later with worsening fevers and tachypnea and was transferred to our hospital for further management the same day. On day 2 of admission, she vaginally delivered a healthy baby girl. She continued to have worsening tachypnea and increasing oxygen requirements postpartum, eventually requiring endotracheal intubation on day 8. She was proned on the following day for refractory hypoxia with improvement in respiratory parameters. One dose of convalescent plasma $(200 \mathrm{~mL})$ was given on day 4 of admission and an $800 \mathrm{mg}$ dose of tocilizumab on day 9 after concomitant bacterial infection was ruled out. She was also started on prophylactic heparin 5,000 units subcutaneously three times a day 2 days after delivery and continued throughout her stay. While intubated, the patient was hemodynamically stable and required only minimal vasopressor medications probably due to sedation effect. The patient's respiratory status started improving and ventilator weaning protocols were initiated. She tolerated supination on day 14 . The patient had persistent low-grade fevers post-delivery with an elevation in her white blood cell in the 12 to $20 \mathrm{k} / \mu \mathrm{l}$ range. Multiple blood cultures were sent that received

accepted

December 21, 2020
September 16, 2020
DOI https://doi.org/

10.1055/s-0041-1723783.

ISSN 2512-9465. were all negative. Her markers of inflammation were elevated initially after intubation with a ferritin of $486.9 \mathrm{ng} / \mathrm{mL}$, Creactive protein of $44.5 \mathrm{mg} / \mathrm{L}$, and sedimentation rate of $75 \mathrm{~mm} / \mathrm{h}$. These markers improved progressively throughout her course. She did not have any laboratory abnormalities suggesting a hypercoagulable state with an initial fibrinogen of $368 \mathrm{mg} / \mathrm{dL}$ that stayed in the normal range throughout her stay. Prothrombin time, activated prothrombin time, and platelet count were also normal on admission and during her hospitalization. Her kidney function was also normal throughout in the 0.36 to $1.07 \mathrm{mg} / \mathrm{dL}$ range. Her D-dimer was elevated to $>20 \mu \mathrm{g} / \mathrm{mL}$ immediately post-delivery but then decreased to $8.2 \mu \mathrm{g} / \mathrm{mL}$ a few days later. Her D-dimer level then gradually increased again, peaking at $>20 \mu \mathrm{g} / \mathrm{mL}$. On day 21 , she complained of abdominal pain. In the setting of persistent fever and leukocytosis, there was concern for an abscess or intraabdominal catastrophe related to her delivery, such as, but not limited to, colonic pseudo-obstruction, infection, uterine involution, or foreign body retention. A computed tomography (CT) scan of the abdomen was therefore obtained that revealed multiple new hypodense splenic infarcts with possible prior hemorrhage into one infarct (-Figs. 1, 2). There were no thromboembolic or infarctions noted on a prior CT chest and abdomen done earlier in the admission. Echocardiogram did not reveal any cardiac thrombi or vegetations that along with the negative blood cultures argued against embolic phenomena. The patient was started on therapeutic dose low molecular weight heparin (LMWH). She was successfully extubated on day 23 and was transitioned to apixaban prior to discharge. She is currently doing well.

Patients with COVID-19 are predisposed to thrombotic complications with cases of pulmonary embolism, deep vein thrombosis, and ischemic strokes reported. ${ }^{1}$ The incidence of thrombotic complications is $\sim 31 \%$ in critically ill patients

(C) 2021. The Author(s).

This is an open access article published by Thieme under the terms of the Creative Commons Attribution License, permitting unrestricted use, distribution, and reproduction so long as the original work is properly cited. (https://creativecommons.org/licenses/by/4.0/)

Georg Thieme Verlag KG, Rüdigerstraße 14, 70469 Stuttgart, Germany 


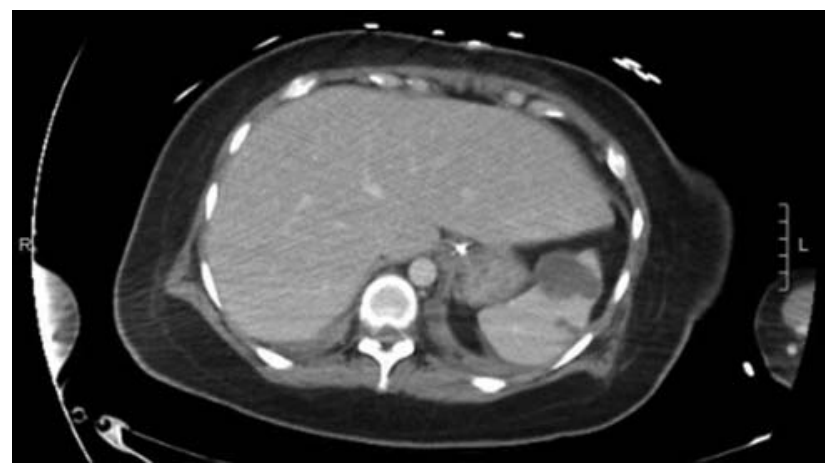

Fig. 1 CT abdomen (axial view) with multiple infarcts in the spleen: largest lesion is $4.1 \mathrm{~cm}$ partially exophytic suspicious for hemorrhage into prior infarct. CT, computed tomography.

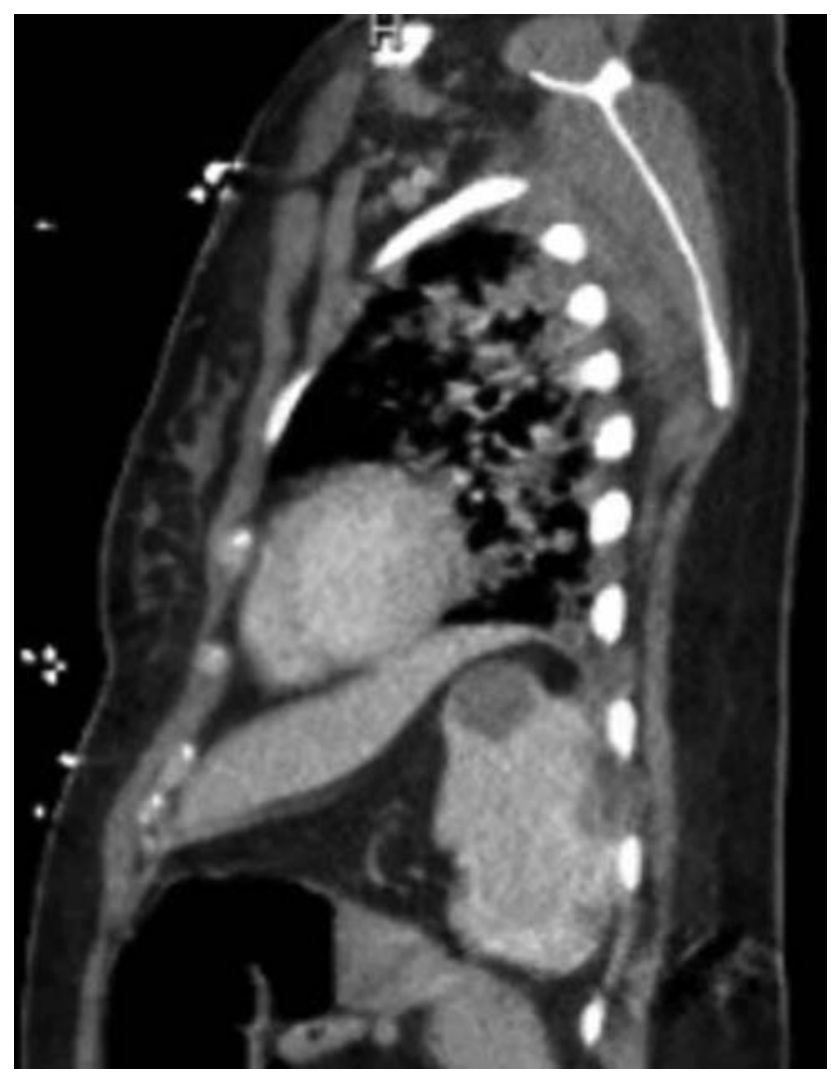

Fig. 2 CT abdomen (sagittal view) with multiple infarcts in the spleen. CT, computed tomography.

with COVID-19. ${ }^{2}$ These complications are thought to be due to the presence of a hypercoagulable state from vascular damage in the setting of sepsis, from the virus itself, and from prolonged immobilization. ${ }^{3}$ Abdominal visceral infarctions have been reported in COVID-19. We found only two case series with four patients in total who had splenic infarctions. Most of the cases had associated thrombosis of the intestines, kidney, or pulmonary vasculature and only two cases of isolated splenic infarction were reported. ${ }^{4-6}$ Pregnancy is a known prothrombotic state as there is an elevation of procoagulant factors like factors II, VII, VIII, XII, and a reduction in anticoagulant factors such as protein $\mathrm{S}$. There is also reduction in fibrinolysis due to increased activity of fibrino- lytic inhibitors. These changes tend to persist up to 8 weeks after delivery. ${ }^{7}$ Splenic infarcts are not a common complication in pregnancy unless the patient has associated conditions like infective endocarditis. ${ }^{8}$ Other potential causes of splenic infarction include malignancy, vasculitis, and red blood cell disorders like sickle cell disease. ${ }^{9}$ Our patient was relatively healthy and did not have a history of obesity, malignancy, autoimmune disorders or vasculitis, atrial fibrillation, and no red blood cell abnormalities were noted on peripheral smear. There was also no personal or family history of hypercoagulability. Patients with splenic infarctions have a varied presentation with fever and left upper quadrant abdominal pain being the most common. Patients may also have left sided chest pain, pleural effusion, hiccups due to diaphragmatic irritation, and abdominal distention. Ultrasound or CT of the abdomen can help with the diagnosis; however, a CT scan is preferred as it provides more information on adjoining organs and blood vessels. The management for splenic infarction involves treatment of the underlying disease state along with aggressive pain control. ${ }^{9}$ When due to a hypercoagulable state, anticoagulation is the mainstay of treatment as was done with our patient. In pregnancy and in the postpartum period, LMWH is preferred and the use of apixaban is off label for splenic infarctions. In our patient, however, we transitioned to apixaban at discharge for patient ease. Further, given her recent pregnancy and COVID-19 infection, we believe that continued anticoagulation was necessary due to an acquired hypercoagulable state. Surgery can be considered in patients with splenic hemorrhage, aneurysm, or abscess but surgery results in a high risk of post splenectomy infections. ${ }^{10}$ Our case is unique in that it describes a patient in her postpartum period with isolated splenic infarctions. Thrombotic complications of COVID-19 are known, but the impact on pregnant and postpartum patients remains unclear. While there are reports of pulmonary embolisms and arterial thrombi in this subgroup, the true incidence is still unknown. ${ }^{11}$ Cases like ours suggest that pregnant patients with COVID-19 might be at a higher risk than nonpregnant COVID-19 patients for thrombotic events. With the high rate of thrombotic complications ${ }^{2}$ and infrequent use of abdominal CT scans, the incidence of splenic and abdominal visceral infarctions is likely underestimated. Guidelines therefore recommend that unless there is an absolute contraindication, all COVID-19 patients should receive chemical venous thromboembolism prophylaxis. ${ }^{12}$ These patients should also be assessed frequently for possible thrombotic events and the need for therapeutic anticoagulation. In pregnant or postpartum patients, there should be a lower threshold to evaluate for thrombotic complications and full-dose anticoagulation.

\section{Authors' Contributions}

All the authors have contributed to this manuscript equally and have seen and approved the final version prior to submission.

\section{Conflict of Interest}

None declared. 


\section{References}

1 Lodigiani C, Iapichino G, Carenzo L, et al;Humanitas COVID-19 Task Force. Venous and arterial thromboembolic complications in COVID-19 patients admitted to an academic hospital in Milan, Italy. Thromb Res 2020;191:9-14

2 Klok FA, Kruip MJHA, van der Meer NJM, et al. Incidence of thrombotic complications in critically ill ICU patients with COVID-19. Thromb Res 2020;191:145-147

3 Barnes GD, Burnett A, Allen A, et al. Thromboembolism and anticoagulant therapy during the COVID-19 pandemic: interim clinical guidance from the anticoagulation forum. [published online ahead of print, 2020 May 21 ]J Thromb Thrombolysis 2020; 50(01):72-81

4 Besutti G, Bonacini R, Iotti V, et al. Abdominal visceral infarction in 3 patients with COVID-19. Emerg Infect Dis 2020;26(08):1926-1928

5 Pessoa M, Lima C, Pimentel AC, Costa Júnior JC, Holanda J. Multisystemic Infarctions in COVID-19: focus on the Spleen. Eur J Case Rep Intern Med 2020;7(07):001747

6 Qasim Agha O, Berryman R. Acute splenic artery thrombosis and infarction associated with COVID-19 disease. Case Rep Crit Care 2020;2020:8880143. Doi: 10.1155/2020/8880143
7 James AH. Pregnancy-associated thrombosis. Hematology (Am Soc Hematol Educ Program) 2009;2009(01):277-285

8 Yuan S-M. Infective endocarditis during pregnancy. J Coll Physicians Surg Pak 2015;25(02):134-139

9 Chapman J, Helm TA, Kahwaji CI. Splenic Infarcts. [Updated 2020 Aug 16]. In: StatPearls [Internet]. Treasure Island (FL): StatPearls Publishing; 2020 Jan-. Accessed January 20, 2021 from: https:// www.ncbi.nlm.nih.gov/books/NBK430902/

10 Salvi PF, Stagnitti F, Mongardini M, Schillaci F, Stagnitti A, Chirletti P. Splenic infarction, rare cause of acute abdomen, only seldom requires splenectomy. Case report and literature review. Ann Ital Chir 2007;78(06):529-532

11 Turan O, Hakim A, Dashraath P, Jeslyn WJL, Wright A, Abdul-Kadir R. Clinical characteristics, prognostic factors, and maternal and neonatal outcomes of SARS-CoV-2 infection among hospitalized pregnant women: a systematic review. Int J Gynaecol Obstet 2020;151 (01):7-16; Epub ahead of print. Doi: 10.1002/ijgo.13329

12 COVID-19 Treatment Guidelines Panel. Coronavirus Disease 2019 (COVID-19) Treatment Guidelines. National Institutes of Health. Accessed November 29, 2020 at https://www.covid19treatmentguidelines.nih.gov/ 